\title{
CALCULATION OF LASER RADIATION DIFFRACTION ON CRYSTAL STRUCTURES BASED ON 3D FOURIER TRANSFORM
}

\author{
O.A. Mossoulina ${ }^{1}$, N.V. Kalinin ${ }^{1}$, M.S. Kirilenko ${ }^{1,2}$ \\ ${ }^{1}$ Samara National Research University, Samara, Russia \\ ${ }^{2}$ Image Processing Systems Institute - Branch of the Federal Scientific Research Centre "Crys- \\ tallography and Photonics" of Russian Academy of Sciences, Samara, Russia
}

\begin{abstract}
In this paper a calculation of $n$-dimensional $(n=1,2,3)$ Fourier transform for various structures, including Gauss-Hermite modes and perovskite crystal cells is made. A significant difference in the diffraction pattern on primitive crystal cells and on perovskite-type crystals is shown.
\end{abstract}

Keywords: diffraction on crystal structures, $n$-dimensional Fourier transform, Hermite-Gaussian modes, perovskites.

Citation: Mossoulina OA, Kalinin NV, Kirilenko MS. Calculation of laser radiation diffraction on crystal structures based on 3D Fourier transform. CEUR Workshop Proceedings, 2016; 1638: 76-82. DOI: 10.18287/1613-0073-20161638-76-82

\section{Introduction}

Crystallography began to be actively developed after the discovery of X-rays by Wilhelm Conrad Roentgen in 1895 [1]. The first X-ray experiment was conducted by Max von Laue in Munich in 1912 [2]. During his work von Laue received experimental evidence that X-rays consisted of high-energy particles; the other data showed that X-rays could be waves. Von Laue suggested that if X-rays were waves, they would have rather short wavelengths.

In 1913 an important event was the discovery made by William Henry and William Lawrence Bragg that X-rays can be used to study positions of atoms inside a crystal, and most importantly, its three-dimensional structure [3]. The inverse lattice is an important mathematical image, and it finds numerous applications in geometric crystallography, in the theory of diffraction and in the structural analysis of crystals, in solid-state physics [4].

At the end of the 20th century a new type of solid substance was discovered, which as well as the crystals has a discrete diffraction pattern, but at the same time has a banned symmetric structure. Such substances are called quasicrystals $[5,6]$. 
Additionally, some hybrid organic-inorganic semiconductor materials, for example with perovskite structure [7], recently acquire great importance in such applied fields as photovoltaics [8,9], where previously crystal silicon was the classical material. The term "perovskite" used for such materials reflects the type of crystal lattice $\mathrm{ABX} 3$, corresponding to the structure of the natural mineral perovskite $\mathrm{CaTiO} 3$, named after Russian mineralogist L.A. Perovsky [10].

Thus, presently not only the existing (natural) crystals are under investigation, but also there is a rapidly developing area of creating new crystals with unusual properties, and also there is an urgent task of investigating the properties of hybrid structures.

The task of calculating the diffraction on the crystal structures can be reduced to performing 3D Fourier transformation [11, 12].

The urgency of implementation of the direct method of calculation of the diffraction pattern on crystal structure is associated with emergence of a wide variety of new artificial crystals - quasicrystals, for which not only three-dimensional, but also twodimensional, and even one-dimensional structures are relevant $[13,14]$.

In this article the calculation of $n$-dimensional $(n=1,2,3)$ Fourier transform for various structures, including Gauss-Hermite modes and perovskite crystal cell.

\section{Theoretical background}

Let us consider the $n$-dimensional Fourier transformation:

$$
F(\mathbf{u})=\mathfrak{I}[f(\mathbf{x})](\mathbf{u})=\int_{R^{n}} f(\mathbf{x}) \exp (-2 \pi i \mathbf{x u}) d^{n} \mathbf{x},
$$

where $f(\mathbf{x})$ is the input function from one variable, $F(\mathbf{u})$ s the resulting output function, $\mathfrak{I}[f(\mathbf{x})](\mathbf{u})$ is Fourier transform operator.

As input functions let us consider the functions of Hermite-Gauss [15], which are a family of own Fourier transform functions $[16,17]$ :

$$
f(\mathbf{x})=\psi_{m}(\mathbf{x}, \sigma)=A \exp \left(-\frac{\mathbf{x}^{2}}{2 \sigma}\right) H_{m}\left(\frac{\mathbf{x}}{\sigma}\right),
$$

where $H_{m}(\mathbf{x})$ - $n$-dimensional Hermite polynomial of $m$-order, $A$ is a normalization coefficient.

And also let us consider a superposition of $n$-dimensional Gaussian beams with which different structures can be approximated, including crystal ones:

$$
f(\mathbf{x})=\sum_{p=1}^{P} c_{p} \exp \left[-\frac{\left(\mathbf{x}-\mathbf{x}_{p}\right)^{2}}{\sigma_{p}^{2}}\right],
$$

where $\mathbf{x}_{p}$ can be regarded as positions of crystal cell nodes, and $\sigma_{p}$ s effective dimensions of respective atoms. 
Note that the Fourier transform of the function (3) to the infinite limits can also be calculated analytically:

$$
F(\mathbf{u})=\sqrt{\pi^{P}} \sum_{p=1}^{P} c_{p} \sigma_{p} \exp \left(-\pi^{2} \sigma_{p}^{2} \mathbf{u}^{2}\right) \exp \left[-2 \pi i \mathbf{x}_{p} \mathbf{u}\right] .
$$

From the expression (4) it is obvious that an interference pattern in the far zone will depend on the positions of nodes as well on the size of atoms.

\section{The results of modeling}

This section presents the results of calculation of the $n$-dimensional $(n=1,2,3)$ Fourier transform for the structures kinds (2) and (3).

Table 1 shows the results of calculation for one-dimensional distributions, Table 2 for two-dimensional, and Table 3 - for three-dimensional. We can see the change in the interference pattern in the diffraction far field depending on the distance between the Gaussian beams, their weight ratios and amounts.

Table 1. The spatial spectrum of one-dimensional structures

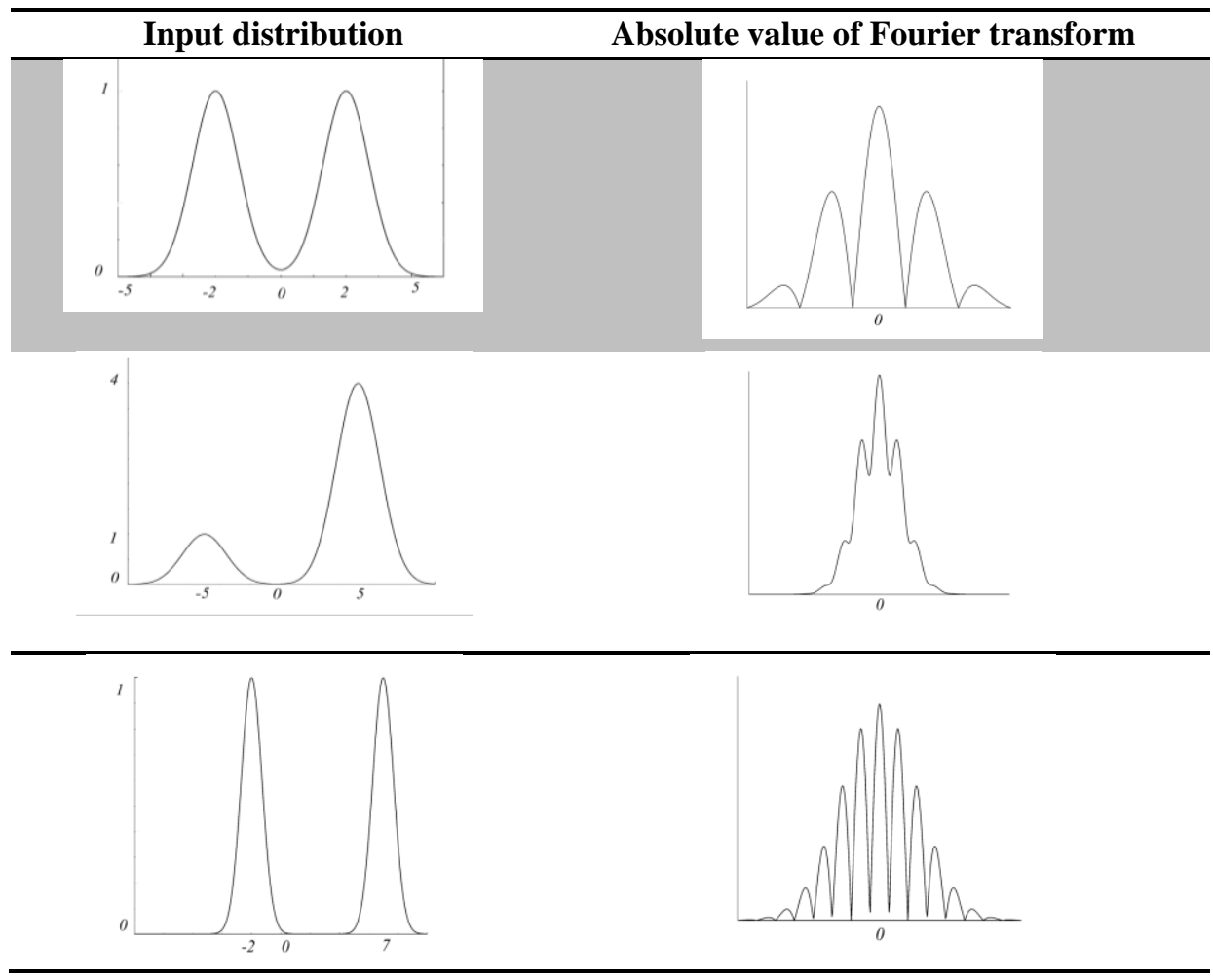


Table 2. The spatial spectrum of two-dimensional structures

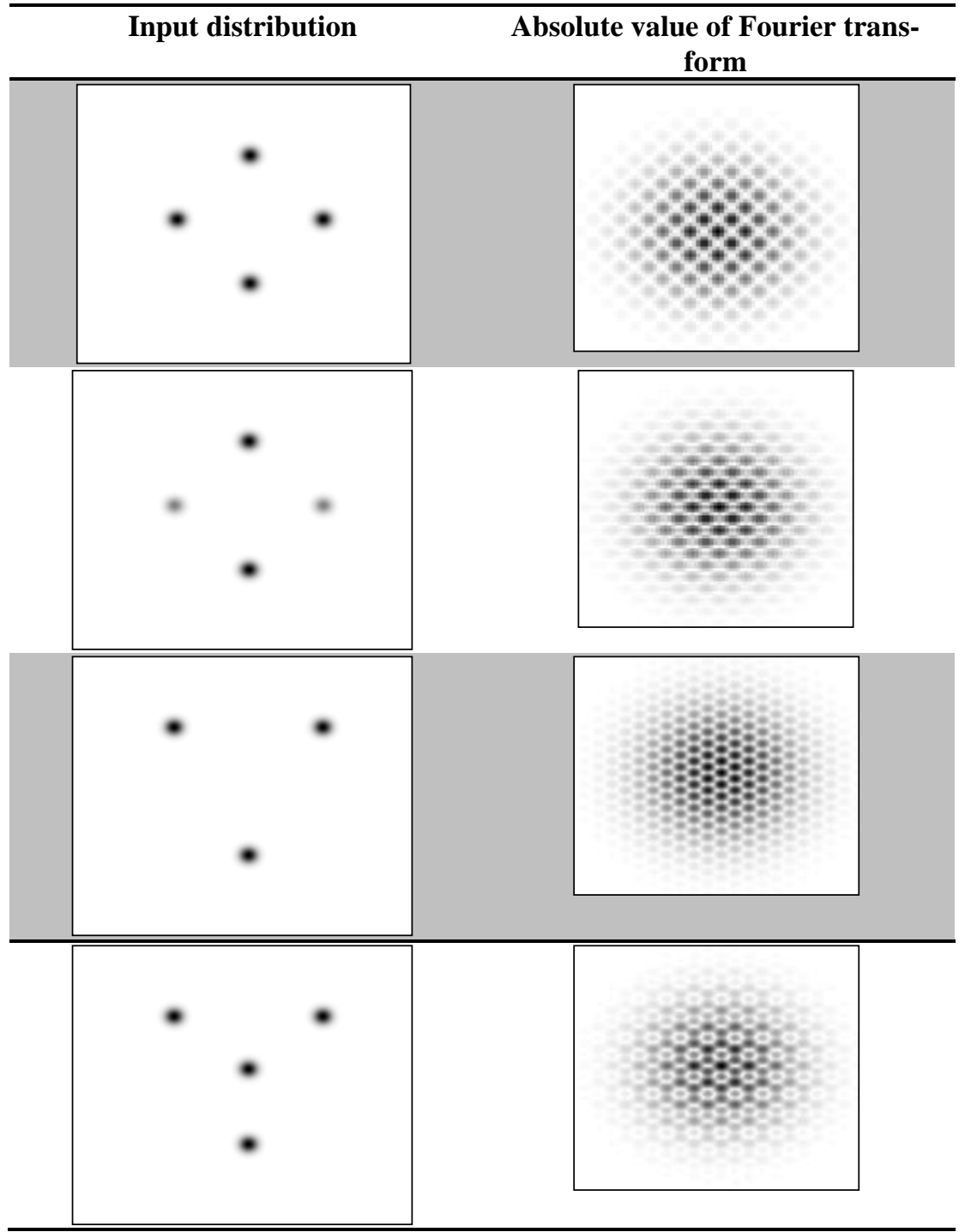

For three-dimensional case visualization was carried out using the software package ParaView.

Fig. 1 shows the three-dimensional Hermite-Gaussian modes with $\mathbf{m}=(2,2,2)$ and $\mathbf{m}=(1,2,3)$ indexes. In the area of spatial spectrum these modes are totally reproduced. Also in the three-dimensional case two primitive crystal cells (Table 3) and the structure of the perovskite type were examined. A significant difference should be noted between the spectral picture of primitive cells and the diffraction on perovskite. 
Table 3. The spatial spectrum of three-dimensional structures

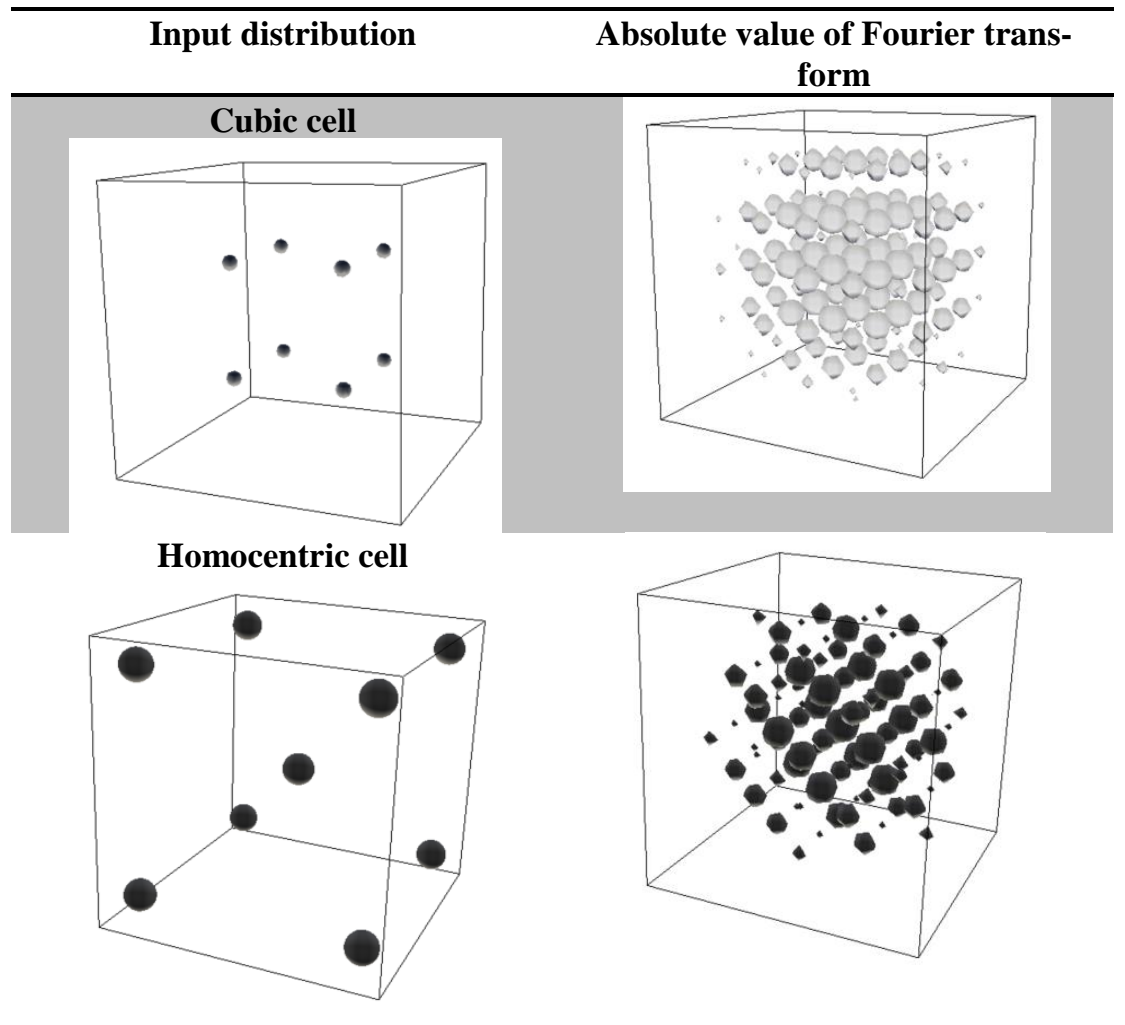

Perovskite cell
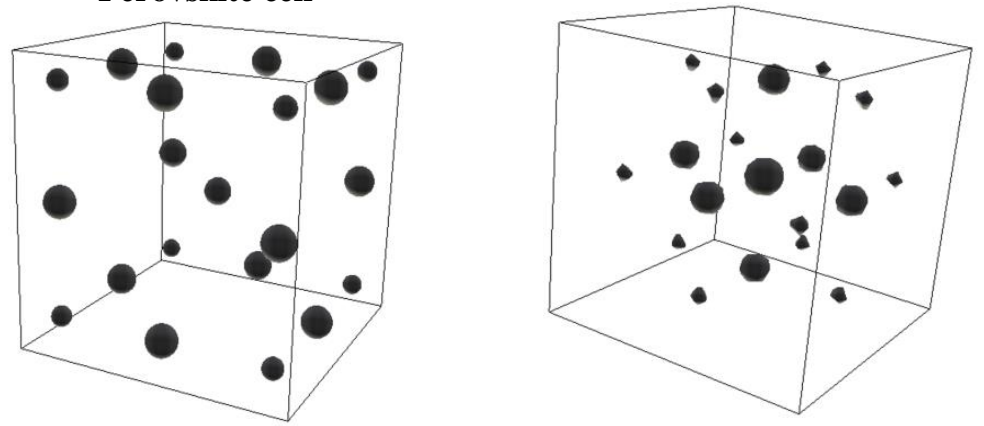

There are many serious packages for studies in the field of crystallography, for example, CRYSTAL, VASP. However, they usually require a certain access and powerful clusters [18].

At the same time there are many open-source software products, such as STEM_CELL and XRayView, allowing to build projections of diffraction patterns for certain crystal structures. 
a)

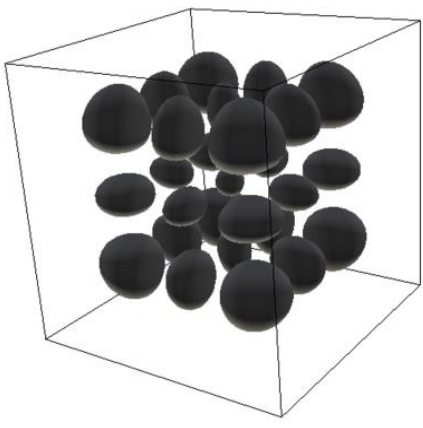

b)

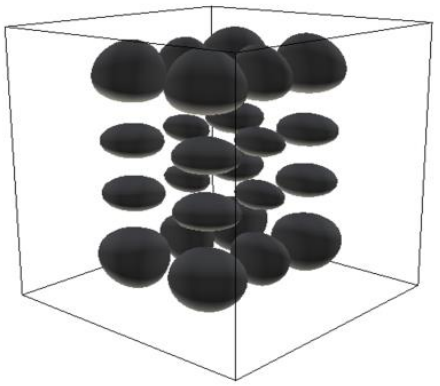

Fig. 1. Distribution of 3D Hermite-Gaussian modes (2) with indexes a) $\mathbf{m}=(2,2,2)$, b) $\mathbf{m}=(1,2,3)$

Figure 2 shows the model of perovskite structure CaTi04, and Fig. 3 - the diffraction patterns in the direction of the axes $(0,0,1)$ and $(1,1,1)$.
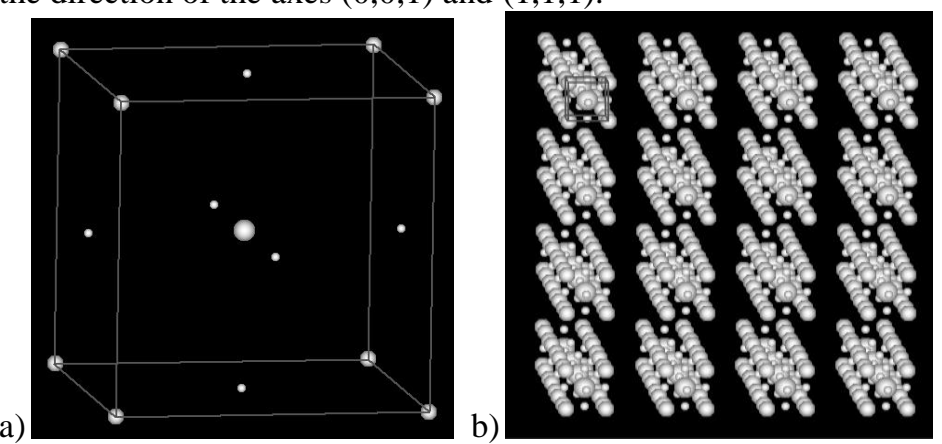

Fig. 2. A model of perovskite structure CaTi04: a) view of a single cell, and b) of the structure obtained by copying.
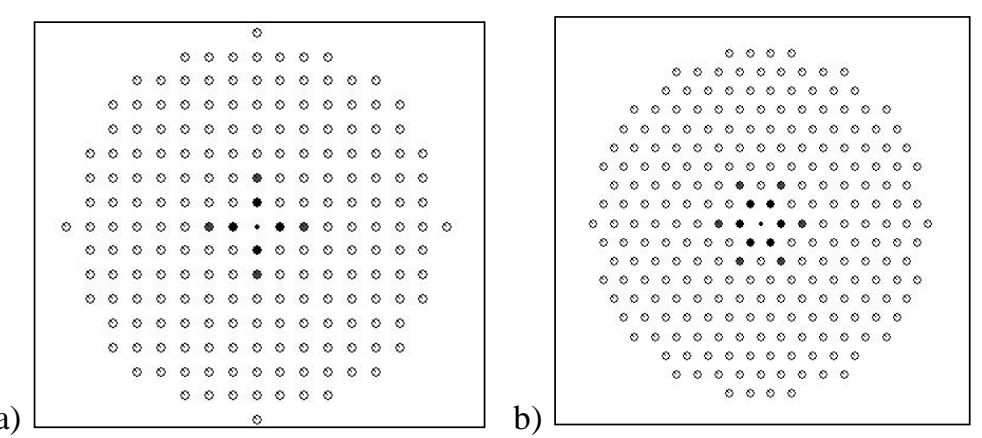

Fig. 3. The diffraction patterns for perovskite $\mathrm{CaTi0} 4$ in the direction of the axes a) $(0,0,1)$ and b) $(1,1,1)$. 


\section{Conclusion}

In this article for approximation of various structures, including crystal ones, superpositions of $n$-dimensional Gaussian beams were examined. Using $n$-dimensional $(n=$ $1,2,3)$ Fourier transform a calculation of the diffraction pattern for different structures was performed, including Gauss-Hermite modes and perovskite crystal cells.

\section{References}

1. Novelline RA, Squire LF. Squire's fundamentals of radiology. Cambridge: Harvard University press, 2004; $638 \mathrm{p}$.

2. Purrington RD. Physics in the nineteenth century, history of science/physics. New Brunswick: Rutgers university press, 1997; 164 p.

3. Bragg WL. The Specular Reflection of X-rays. Nature, 1912; 90: 410-410.

4. Katsnelson AA, Krinchik GS. Solid State Physics (Special practical). Moscow: Publisher of MSU, 1982; 304 p. [in Russian]

5. Steinhardt DL, Steinhardt PJ. Quasicrystals: A New Class of Ordered Structures. Physical Review Letters, 1984; 53: 2477-2480.

6. Lidin S. The Discovery of Quasicrystals. Scientific Back-ground on the Nobel Prize in Chemistry, 2011; 11 p.

7. Stranks SD, Burlakov VM, Leijtens T, Ball JM, Goriely A, Snaith HJ. Recombination kinetics in organic-inorganic perovskites: excitons, free charge, and subgap states. Phys. Rev. App 1., 2014; 2(3): 034007.

8. Snaith HJ. Perovskites: the emergence of a new era for low-cost, high-efficiency solar cells. J. Phys. Chem. Lett., 2013; 4: 3623-3630.

9. Hodes G. Perovskite-based solar cells. Science, 2013; 342: 317-318.

10. L. J. Schmidt. Tracking down the truth of perovski. 38th Rochester Mineralogical Symp. Program Notes, 2011; 31-32.

11. Kharitonov SI, Volotovskiy SG, Khonina SN, Kazanskiy NL. A differential method for calculating x-ray diffraction by crystals: the scalar theory. Computer Optics, 2015; 39(4): 469-479. DOI: 10.18287/0134-2452-2015-39-4-469-479.

12. Kharitonov SI, Kazansky NL, Volotovskiy SG, Khonina SN. Calculating x-ray diffraction on crystals by means of the differential method. Proc. SPIE, 2016; 9807: 98070V-10 p.

13. Pawley GS. Unit-cell refinement from powder diffraction scans. J. Appl. Crystallogr., 1981; 14(6): 357-361.

14. Le Bail A, Duroy H, Fourquet JL. Ab-initio structure determination of LiSbWO 6 by Xray powder diffraction. Materials Research Bulletin, 1988; 23(3): 447-452.

15. Yariv A. Introduction to Optical Electronics. Moscow: "Vysshaya shkola" Publisher, 1983; 400 p. [in Russian]

16. Kotlyar VV, Khonina SN, Soifer VA. Generalized Hermite beams in free space. Optik, 1998; 108(1): 20-26.

17. Khonina SN, Kotlyar VV, Soifer VA. Self-reproduction of multimode Hermite-Gaussian beams. Technical Physics Letters, 1999; 25(6): 489-491.

18. Kresse G., Furthmuller J. Efficiency of ab-initio total energy calculations for metals and semiconductors using a plane-wave basis set. Comput. Mater. Sci., 1996; 6: 15-50. 